\title{
Prevalencia de los factores de riesgo perinatales en los Trastornos Generalizados del Desarrollo*
}

\author{
Prevalence of Perinatal Risk Factors in the \\ Pervasive Developmental Disorders
}

Enviado: septiembre 30 de 2010 | Revisado: mayo 20 de 2011 | Aceptado: diciembre 12 de 2011

\author{
SANTIAGO LÓPEZ GÓMEZ ** \\ ROSA MARÍA RIVAS TORRES *** \\ Eva María TABOADA ARES **** \\ Universidad de Santiago de Compostela, España
}

\section{RES U MEN}

Los Trastornos Generalizados del Desarrollo (TGD) son perturbaciones graves y generalizadas que afectan áreas centrales del desarrollo (DSM-IV-TR). Se propone que el período perigestacional aglutina una serie de factores de riesgo que influyen y condicionan el desarrollo normal del feto. El objetivo de este artículo fue estudiar la presencia de riesgos durante el desarrollo perinatal, considerando las respuestas de 93 madres con hijos que presentan un Trastorno Generalizado del Desarrollo a un autoinforme estructurado, tal como es el caso del trastorno autista, trastorno de Asperger y TGD-no especificado. Del análisis de las respuestas al autoinforme se han encontrado diferencias significativas entre los grupos de TGD en la dimensión pregestacional -malnutrición/anorexia e hipertensión-, en la perigestacional -malnutrición/anorexia y problemas con el líquido amniótico- y en la psicosocial -género no deseado del bebé-.

Palabras clave autores:

Trastornos generalizados del desarrollo, TGD, trastorno autista, trastorno de

Asperger, TGD-NE, etiología, riesgo perinatal.

Palabras clave descriptores:

Psicología de la salud, promoción y prevención, desarrollo.

\section{A B S T R A C T}

Pervasive Developmental Disorders (PDDs) are severe and pervasive disturbances affecting central areas of development (DSM-IV-TR). It is proponed that the perigestational period encompasses a number of risk factors that

Para citar este artículo: López, S., Rivas, R. M. \& Taboada, E. M. (2012). Prevalencia de los factores de riesgo perinatales en los Trastornos Generalizados del Desarrollo. Universitas Psychologica, 11(3), $875-883$.

\footnotetext{
Artículo de investigación.

** Profesor del Departamento de Psicología Evolutiva y de la Educación.E-mail: slopezg@udc.es

*** Profesora y Directora del Departamento de Psicología Evolutiva y de la Educación. E-mail: rosa.rivas@usc.es

****** Profesora del Departamento de Psicología Evolutiva y delaEducación.E-mail: evamaria.taboada@usc.es
} influence and affect normal fetal development. The aim of this paper was to study the presence of risks during the perinatal development, considering the responses of 93 mothers of children with a pervasive developmental disorder -autistic disorder, Asperger disorder and PDD-NOS- to a structured self-report. We found significant differences among the PDD groups in the pregestational -malnutrition/anorexia and hypertension-, in the perigestational - malnutrition/anorexia and problems with the amniotic fluid-, and in the psychosocial -unwanted gender baby-dimensions from the analysis of the responses.

Key words authors:

Pervasive Developmental Disorders, PDD, Autistic Disorder, Asperger Disorder, PDD-NOS, aetiology, perinatal risk.

Key words plus:

Health Psychology, Promotion and Prevention, Development. 


\section{Introducción}

Los Trastornos Generalizados del Desarrollo (TGD) se caracterizan por un conjunto de alteraciones neuropsicológicas graves, generalizadas y heterogéneas que interfieren en varios aspectos del desarrollo -social, comunicativo y conductual- (American Psychiatric Association [APA], 2002). Dichas alteraciones afectan habilidades relacionadas con la interacción social, con el lenguaje y la comunicación y con el nivel comportamental, mostrando intereses y actividades que se caracterizan por ser restringidas y estereotipadas. Se incluyen como TGD: el trastorno autista (TA), el trastorno de Rett (TR), el trastorno desintegrativo infantil (TD), el trastorno de Asperger (TAs) y el trastorno generalizado del desarrollo no especificado (TGD-NE).

Estos trastornos tienen un origen desconocido, si bien hoy en día parece que cobran fuerza los factores neurobiológicos como eje de su etiología. Aparentemente, hay una marcada influencia de naturaleza biológica (genética, neurológica, metabólica, etc.) y ambiental, procedentes tanto del propio entorno como de la constitución personal, que estarían interactuando, junto con un número de factores aún desconocidos, para desencadenar los TGD (Álvarez, 2007; Bayés et al., 2005; López, Rivas \& Taboada, 2008, 2009; MacLean et al., 1999; Rivas, López \& Taboada, 2009; Szatmari, 2003).

Sin duda, los estudios realizados en los últimos años definen a los TGD, en un sentido amplio, como resultado de alteraciones en el desarrollo de diversas funciones del Sistema Nervioso Central (SNC) (Fisher \& Happé, 2005; Folstein, 1999; Zwaigenbaum et al., 2005). Ahora bien, este planteamiento considera también la coexistencia de multiplicidad de factores, tanto ambientales como hereditarios, con sus diversas formas de conjugarse y, por lo tanto, de manifestarse (Cook, 2001; Croen, Grether, Hoogstrate \& Selvin, 2002; López et al., 2009; Stein, Weizman, Ring \& Barak, 2006; Wilkerson, Volpe, Dean \& Titus, 2002).

En esta línea, se han identificado en el período anterior al embarazo - pregestacional- una serie de factores de riesgo como posibles responsables de complicaciones posteriores en el desarrollo. Entre ellos se destacan: 1) la edad de la madre (Fraser, Brockert \& Ward, 1995; Orvos et al., 1999), 2) las características físicas maternas y paternas (Pritchard, Sutherland \& Carr-Hill, 1983) o 3) el peso de la madre, ya que este puede condicionar, si se sitúa fuera de los índices de normalidad, el correcto desarrollo fetal. A su vez, un historial reproductivo adverso, puede suponer también un riesgo frente a un nuevo embarazo (Saisto, Ylikorkala \& Halmesmaki, 1999).

Al mismo tiempo, se ha hipotetizado que el embarazo, el parto, e incluso las complicaciones neonatales pueden actuar, desde diversos frentes, incrementando el riesgo del autismo y de los demás TGD, o bajo otro supuesto, interactuando conjuntamente con los determinantes genéticos y de esta manera exacerbar el riesgo en ese período crítico del proceso de desarrollo.

En este sentido, el periodo de tiempo que dura la gestación -perigestacional-aglutina una serie de factores de riesgo que pueden llegar a influenciar y condicionar el crecimiento y desarrollo normal del feto. Entre ellos pueden destacarse:

- El consumo de sustancias químicas por parte de la madre -tabaco, alcohol, drogas, fármacos teratógenos- puede suponer un riesgo en el crecimiento y resultado fetal, asociándose a una amplia gama de alteraciones en el desarrollo, como el bajo peso, los trastornos psicomotrices, sensoriales y de conducta, las alteraciones cerebrales y los daños neurológicos, entre otros (Astley \& Clarren, 2001; Kesmodel, Wisborg, Olsen, Brink \& Secher, 2002; Lester et al., 2002; Ribas-Fito et al., 2003; Ventura, Hamilton, Mathews \& Chandra, 2003; Wideroe, Vik, Jacobsen \& Bakketeig, 2001).

- El peso ganado por la madre a lo largo del embarazo correlaciona con el peso final del bebé (Shapiro, Sutija \& Bush, 2000). Las situaciones tanto de sobrepeso como de malnutrición materna van a condicionar el término de la gestación y, a la vez, incrementar la mortalidad infantil, los partos prematuros y las alteraciones en el desarrollo neurológico y cognitivo (American College of Obstetrician and Gynecologist [ACOG], 1993).

- Las condiciones médicas maternas y/o los acontecimientos médico-obstétricos previos a la 
gestación y durante la misma -sangrados vaginales, edemas, hipertensión, trastornos endocrinos, problemas cardiacos, infecciones- son de vital importancia para el óptimo desarrollo del neonato. Estas circunstancias van a condicionar su nacimiento y su estado de salud o de enfermedad (Correa, Botto, Liu, Mulinare \& Erickson, 2003; Faden, Hanna \& Graubard, 1997; Ribas-Fito et al., 2003).

- Los accidentes o lesiones durante el embarazo pueden asociarse a diversos daños de distinta consideración suponiendo, en algunos casos, riesgos para la madre y para el feto -muerte fetal anteparto, infecciones, desgarros, lesiones en órganos, falta de oxígeno, entre otros-.

Por otra parte, distintos factores de naturaleza psicosocial pueden contribuir a potencializar los riesgos perinatales y a su vez alterar el posterior desarrollo del niño. En este sentido, los acontecimientos y situaciones estresantes vividas por la madre, junto con la realización de trabajo físico y mental duro y agotador durante el embarazo pueden provocar prematurez, bajo peso, alteraciones neurológicas diversas, trastornos comportamentales, problemas en el desarrollo, en el control emocional y en la motricidad (Huizink, Robles, Mulder, Visser \& Buitelaar, 2002; Lobel, 1994; Lou et al., 1994).

En esta línea, diversos estudios han analizado la relación entre algunos riesgos y factores de orden perinatal y los TGD, comparando el desarrollo gestacional de estos sujetos con sus hermanos o con un grupo control (Baron-Cohen \& Bolton, 1994; Bolton et al., 1997; Deykin \& MacMahon, 1980; Finegan \& Quarrington, 1979; Gillberg \& Gillberg, 1983; Iverson \& Wozniak, 2007; Juul-Dam, Townsend \& Courchesne, 2001; Matsuishi et al., 1999; Stein et al., 2006; Toth, Dawson, Meltzoff, Greenson \& Fein, 2007; Wilkerson et al., 2002; Zwaigenbaum et al., 2002). Sin embargo, hasta el momento, los factores encontrados no identifican de manera consistente una relación directa entre los TGD y un historial de riesgo perinatal, de manera que los factores de riesgo específicos hallados no permiten una interpretación clara de los procesos patológicos implicados.

En el presente estudio, se analizan los posibles riesgos durante el desarrollo perinatal en una muestra de madres con hijos que presentan TGD. Se valoran sus respuestas en un autoinforme estructurado sobre los factores de riesgo asociados al embarazo. El análisis del posible impacto diferencial de los riesgos perinatales en los TGD no se realiza comparativamente con un grupo control, como viene siendo habitual en las investigaciones, sino, y de manera novedosa en este artículo, entre los distintos tipos de TGD.

\section{Método}

\section{Participantes}

En la investigación participaron un total de 93 madres biológicas de hijos con un diagnóstico de TGD, según los criterios de los DSM-IV-TR (APA, 2002) -68 con trastorno autista (TA), 6 con trastorno de Asperger (TAs) y 19 con TGD no especificado (TGD-NE)-. Todas las madres pertenecían a las distintas asociaciones de autistas de Galicia (España). El diagnóstico de los subtipos de TGD se realizó siempre por equipos multidisciplinares pertenecientes a la Federación Autismo-Galicia, con gran experiencia y formación en TGD.

\section{Materiales}

La recogida de información sobre la presencia de riesgos perinatales se realizó con el Cuestionario Materno de Riesgo Perinatal (CMRP), un autoinforme estructurado, diseñado ad hoc. Está constituido por dos grupos, en uno se incluyen las dimensiones relativas al desarrollo perinatal: preperigestacional, intraparto y neonatal, en el otro, las de naturaleza psicosocial, esto es, las variables psicosociales y las sociodemográficas. A través de 70 ítems y del grupo de identificadores familiares de carácter sociodemográfico, se contemplan la presencia o ausencia de 40 factores de riesgo pre y perigestacionales (López, 2004; López et al., 2008). Los autoinformes estructurados han demostrado una gran fiabilidad en la recogida de información retrospectiva sobre el periodo del embarazo. Coinciden, de manera casi exacta, con una correlación superior a 0.9 , con los datos que contienen sus historiales 
médicos y muestran también altas correlaciones con otras medidas neonatales, como las ofrecidas por el Apgar, por esto han sido utilizadas, con gran acierto, en múltiples investigaciones (Dean, Gray \& Anderson, 1996; Gray, Dean \& Rattan, 1987; Gray, Dean, Rattan \& Bechtel, 1988).

\section{Procedimiento}

El CMRP se aplicó de manera individual a madres biológicas pertenecientes a las asociaciones de autismo de Galicia (España). Todas ellas tenían un hijo con un diagnóstico de TGD. Una vez cubierto el Cuestionario tanto en el grupo experimental como en el control de manera voluntaria y anónima se procedió a la codificación y al análisis de los datos.

\section{Resultados}

Se realizó un análisis descriptivo de las puntuaciones medias y desviaciones típicas, así como un estudio de distribución de frecuencias y porcentajes de los distintos factores e ítems -agrupados en las dimensiones anteriormente comentadas-a los que hace referencia el citado Cuestionario. El objetivo del análisis descriptivo fue conocer cómo se manifestaban los distintos riesgos perinatales en las diferentes tipologías de los TGD encontrados en la muestra. Para determinar la existencia de diferencias significativas entre los distintos grupos, se realizó un análisis comparativo de las frecuencias, utilizando el estadístico chi cuadrado para cada uno de los ítems del CMRP.

Los datos sociodemográficos referidos a la edad y orden de nacimiento del hijo, edad de la madre en el embarazo, edad actual de la madre y del padre, así como nivel de estudios y categoría profesional, se recogen en la Tabla 1.

La comparación de frecuencias de los distintos subgrupos de TGD mediante el estadístico chi cuadrado, reflejó la existencia de diferencias significativas en dos de los ítems incluidos en la dimensión pregestacional: la desnutrición/anorexia anterior al embarazo y la hipertensión arterial anterior al embarazo (véase Tabla 2).

La puntuación significativa en el factor malnutrición/anorexia anterior al embarazo $\left[\chi^{2}(2)=8.378\right.$, $p<0.05$ ] confirmó la existencia de una relación entre la presencia de un trastorno generalizado del desarrollo y la desnutrición/anorexia pregestacional. Las máximas puntuaciones entre los tres grupos se obtuvieron en el TAs, con una frecuencia de 1 (16.7\%), sobre una frecuencia esperada de 0.1 y en el TGD-NE, con una frecuencia también de 1 (5.3\%), de una frecuencia esperada de 0.4. Sin embargo, el grupo con TA destacó por la ausencia completa de problemas relacionados con la alimen-

TABLA 1

Descriptivos sociodemográficos de los grupos con TGD

\begin{tabular}{lcccccr}
\hline & \multicolumn{2}{c}{ TA } & \multicolumn{2}{c}{ TAs } & \multicolumn{2}{c}{ TGD-NE } \\
\cline { 2 - 7 } & M & DE & M & DE & M & SD \\
\hline Edad del hijo & 169.79 & 88.52 & 125 & 62.81 & 171.68 & 85.93 \\
Orden de nacimiento del hijo & 1.54 & 0.74 & 1.16 & 0.4 & 1.57 & 1.01 \\
Edad de la madre en el embarazo & 27.58 & 6.1 & 26.33 & 3.5 & 27.78 & 5.25 \\
Edad actual de la madre & 42.17 & 7.56 & 37 & 5.93 & 41.66 & 8.79 \\
Edad actual del padre & 44.85 & 7.64 & 38.2 & 7.04 & 44.26 & 10.58 \\
Nivel de estudios de la madre (1-4) & 3 & 1.93 & 3 & 1.78 & 1.93 & 1.43 \\
Nivel de estudios del padre (1-4) & 3.06 & 0.83 & 3 & 1 & 2.62 & 0.8 \\
Categoría profesional de la madre (1-6) & 3.25 & 1.93 & 3 & 1.78 & 1.93 & 1.43 \\
Categoría profesional del padre (1-6) & 4.45 & 0.83 & 3 & 1 & 2.62 & 0.8 \\
\hline
\end{tabular}

Nota. Los valores entre paréntesis representan los diferentes niveles educativos (1-4) y profesionales (1-6) que se han estudiado. $\mathrm{TA}=$ Trastorno Autista; TAs = Trastorno de Asperger; TGD-NE = TGD-No Especificado

Fuente: elaboración propia. 
TABLA 2

Resultados comparativos significativos en las dimensiones y factores de riesgo entre los distintos grupos

\begin{tabular}{|c|c|c|c|c|c|c|c|}
\hline & \multicolumn{2}{|c|}{$\begin{array}{l}\text { T. Autista } \\
(\mathrm{N}=68)\end{array}$} & \multicolumn{2}{|c|}{$\begin{array}{c}\text { TAs } \\
(N=6)\end{array}$} & \multicolumn{2}{|c|}{$\begin{array}{l}\text { TGD-NE } \\
(\mathrm{N}=19)\end{array}$} & \multirow[t]{2}{*}{$\chi^{2}$} \\
\hline & $\mathrm{N}$ & $\%$ & $\mathrm{~N}$ & $\%$ & $\mathrm{~N}$ & $\%$ & \\
\hline Malnutrición previa / anorexia & & & & & & & $8.378^{*}$ \\
\hline No & 68 & 100 & 5 & 83.3 & 18 & 94.7 & \\
\hline Sí & - & - & 1 & 16.7 & 1 & 5.3 & \\
\hline Hipertensión previa & & & & & & & $6.575^{*}$ \\
\hline No & 67 & 98.5 & 5 & 83.3 & 19 & 100 & \\
\hline Sí & 1 & 1.5 & 1 & 16.7 & - & - & \\
\hline Malnutrición / anorexia durante el embarazo & & & & & & & $7.961 * *$ \\
\hline No & 68 & 100 & 6 & 100 & 17 & 89.5 & \\
\hline Sí & - & 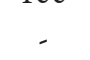 & - & 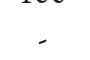 & 2 & 10.5 & \\
\hline Problemas con el líquido amniótico & & & & & & & $14.658^{* * *}$ \\
\hline No & 68 & 100 & 5 & 83.3 & 19 & 100 & \\
\hline Sí & - & - & 1 & 16.7 & - & - & \\
\hline Sexo no deseado del bebé & & & & & & & $15.283 *$ \\
\hline Sí deseado & 32 & 47.1 & 2 & 33.3 & 10 & 52.6 & \\
\hline Indiferente & 7 & 10.3 & 1 & 16.7 & 2 & 10.5 & \\
\hline No deseado & - & - & 1 & 16.7 & - & - & \\
\hline Sexo desconocido hasta nacer & 29 & 42.6 & 2 & 33.3 & 7 & 36.8 & \\
\hline
\end{tabular}

$* p<0.05 ; * * p<0.01 ; * * * p \leq 0.001$

Fuente: elaboración propia.

tación en la madre previa a la gestación, de una frecuencia esperada de 1.5 .

La hipertensión anterior al embarazo resultó también ser significativa en el análisis de chi cuadrado $\left[\chi^{2}(2)=6.575, p<0.05\right]$. En este caso, el $98.5 \%$ de las madres pertenecientes al grupo con TA, de una frecuencia esperada de 66.5, afirmaron haber padecido hipertensión anterior al embarazo; solo el $1.5 \%$, de una frecuencia esperada de 1.5, declaró no haberla tenido. En el grupo del TAs, el $16.7 \%$, de una frecuencia esperada de 0.1 , indicó que tuvo hipertensión anterior, mientras que el $83.3 \%$, de una frecuencia esperada de 5.9, no la tuvo. En el último grupo, el de madres con hijos con TGD-NE, ninguna de ellas, de una frecuencia esperada de 0.4, sufrió de hipertensión anterior al embarazo.

En el resto de los ítems incluidos en la dimensión pregestacional no se obtuvieron diferencias significativas entre los tres subgrupos que conforman la muestra.
En la dimensión perigestacional se observaron diferencias estadísticamente significativas al comparar las frecuencias de los grupos, en dos ítems: malnutrición/anorexia durante el embarazo $\left[\chi^{2}(2)=7.961, p<0.01\right]$ y problemas con el líquido amniótico $\left[\chi^{2}(2)=14.658, p<0.001\right]$, ambos incluidos en el historial de enfermedades durante la gestación.

En cuanto a la desnutrición/anorexia durante el embarazo se comprobó que ninguna madre de los grupos con TA y con TAs afirmó haber tenido problemas con la alimentación durante su embarazo, con una frecuencia esperada de 1.5 y de 0.1 , respectivamente. En el grupo con TGD-NE, el $10.5 \%$, de una frecuencia esperada de 0.4 , manifestaron haber tenido problemas de alimentación durante el embarazo. En el resto de las muestras de los tres subgrupos, la anorexia, la desnutrición o los problemas de alimentación no se hicieron notar durante el desarrollo gestacional. 
Los problemas con el líquido amniótico se manifestaron de forma exclusiva en el $16.7 \%$ del grupo con TAs, de una frecuencia esperada de 0.1. En el grupo con TA no se encontró dicho problema, aunque su frecuencia esperada era de 0.7 , al igual que tampoco se observó en el grupo con TGD-NE, con una frecuencia esperada de 0.2 .

Los restantes ítems agrupados en la dimensión perigestacional no reflejaron un valor significativo suficiente, por ello se confirma la independencia de los grupos del TGD en dichos ítems.

Al aplicar el estadístico chi cuadrado para comparar los tres grupos de la muestra en la dimensión intraparto, de los siete ítems que describen dicha dimensión no se encontró alguno con valores significativos, por lo que no se puede deducir relación entre los tres grupos estudiados. Tampoco se han obtenido diferencias significativas en la dimensión neonatal en los seis ítems valorados. Por esto, subrayamos la independencia de los grupos.

La dimensión psicosocial estaba compuesta por ocho ítems en el CMRP. De ellos, un único ítem -sexo deseado del bebé- obtuvo un valor de significatividad en chi cuadrado $\left[\chi^{2}(6)=15.283\right.$, $p<0.05]$.

En el grupo con TA, el $47.1 \%$ de las madres -de una frecuencia esperada de 32.2- afirmaron alegrarse al conocer el sexo de su bebé. De este mismo subgrupo, el $10.3 \%$-de una frecuencia esperada de 7.3- manifestaron su indiferencia ante el sexo del bebé que esperaban, y ninguna -de una frecuencia esperada de 0.7-indicó haber mostrado tristeza. Sin embargo, un alto número de madres de este grupo con TA (42.6 \%) -de una frecuencia esperada de 27.8 - no conocieron el sexo de su bebé hasta el momento de su nacimiento. En el grupo de madres de hijos con TAs, el $33.3 \%$ de las madres -de una frecuencia esperada de 2.8- manifestó haber sentido alegría; y en un $16.7 \%$ de los casos indiferencia o tristeza -de una frecuencia esperada de 0.6 y 0.2 respectivamente-; mientras que el $33.3 \%$ afirmó no conocer el sexo de su bebé hasta que tuvo lugar su nacimiento -de una frecuencia esperada de 2.5-. En relación al grupo con TGD-NE, se obtuvo que el $52.6 \%$ de las madres -de una frecuencia esperada de 9.0- se sintió alegre al conocer el sexo del bebé; el $10.5 \%$-de una frecuencia esperada de 2.0 - se mostró indiferente y ninguna experimentó tristeza como reacción emocional -de una frecuencia esperada de 0.2-. En un porcentaje similar al grupo anterior, el $36.8 \%$-de una frecuencia esperada de 7.8 - no conoció el sexo del bebé hasta que se produjo su nacimiento.

La dimensión sociodemográfica, compuesta por cuatro factores (profesión de la madre, nivel de estudios de la madre, profesión del padre y nivel de estudios del padre), se mostró independiente al realizar el análisis de los subgrupos en chi cuadrado y valorar su nivel de significación alcanzado. Ninguno de los ítems reflejó un valor significativo para esta dimensión.

\section{Discusión}

El análisis diferencial de riesgos maternos perinatales entre los TGD es un tema poco investigado. En la literatura científica contemplada para la investigación no se han encontrado estudios que describan comparativamente los riesgos maternos perinatales entre los tipos de TGD. Por el contrario, es extenso el número de investigaciones que analizan la relación entre los riesgos perinatales y los TGD (trastorno autista fundamentalmente) con sus hermanos o con un grupo control, tal y como se expone en páginas anteriores.

Los problemas relacionados con la alimentación -desnutrición/anorexia-, tanto en la dimensión pregestacional como perigestacional, marcan diferencias significativas entre los subgrupos destacándose como importantes factores de riesgo perinatal. Es necesario tomar en consideración también la hipertensión como enfermedad previa a la gestación. Diversos estudios, aunque centrados en el momento perinatal (Deykin \& MacMahon, 1980; Gillberg \& Gillberg, 1983), valoran la asociación entre la presencia de hipertensión y el $\mathrm{TA}$, no encontrando diferencias significativas en comparación con un grupo control o con hermanos de estos niños.

En la dimensión perigestacional, además de los problemas de alimentación, destacan también los problemas con el líquido amniótico que, por otro 
lado, se muestran con una muy baja frecuencia para ambos factores en los tres subgrupos estudiados en la muestra y siempre con la presencia superior en el TGD-NE. Finegan y Quarrington (1979) y Gillberg y Gillberg (1983) habían encontrado también una presencia marcada de problemas con el líquido amniótico en niños con TA.

En el resto de los factores estudiados, atendiendo a la gran homogeneidad de los datos, se puede decir que los tres subgrupos se comportan como una misma muestra poblacional no evidenciando diferencias estadísticamente significativas.

Por lo que respecta a la dimensión psicosocial, se reafirma la diferencia entre los subgrupos en cuanto al ítem del sexo deseado del bebé. Así, las situaciones de aceptación se constataron en el subgrupo del TGD-NE y del TA, frente a una mayor indiferencia y rechazo en el TAs. Si bien, un alto número de madres con hijos con TA y TGD-NE manifestaron no haber llegado a conocer el sexo del bebé hasta que nació. Este hecho se podría relacionar con las investigaciones de Finegan y Quarrington (1979) y de Levy, Zoltak y Saelens (1988), quienes encontraron una presentación anormal en el parto en sus estudios con niños con TA. Esta circunstancia tal vez resulta indicativa de una mala posición en el útero durante la etapa de desarrollo fetal que provoca el desconocimiento del sexo del bebé al no poder ser visto en los controles médicos realizados a la madre gestante. Aunque también surgen otras posibles explicaciones: simplemente que no se deseara conocer el sexo del bebé o que los citados controles no hayan tenido lugar, con el consiguiente riesgo que esto supone.

En las dimensiones intraparto, neonatal y sociodemográfica no se observaron diferencias significativas entre los subgrupos con TA, TAs o TGD-NE, en los ítems estudiados. Este resultado asegura que se trata de una misma muestra en su conjunto, en cuanto a los factores de riesgo que dichas dimensiones agrupan.

Como conclusión, se indica que es necesario reflexionar sobre la realización de nuevos estudios con criterios de valoración unificados, en los cuales se deberán considerar otras variables no contempladas, como las referidas al desarrollo y a las adquisiciones del niño en sus primeros años, las relativas a su $\mathrm{CI}$, la presencia de riesgos perinatales en el historial familiar, como también el estado mental de los padres tanto en la actualidad como en su historia individual y familiar. Al mismo tiempo, dado que el grupo que compone la muestra es de tamaño reducido, sobre todo para los grupos con TAs y TGD-NE, los resultados no deben llevar a conclusiones definitivas sino provisionales, en espera de ser confirmados con poblaciones más amplias. Ahora bien, se deben considerar los que muestran estadísticas significativas, pues suponen un avance en el establecimiento de un método de estudio que puede ser interesante para valorar riesgos perinatales frente a diversas patologías y, en concreto, frente a los TGD.

\section{Referencias}

Álvarez, E. (2007). Trastornos del espectro autista. Revista Mexicana de Pediatría, 74(6), 269-276.

American College of Obstetrician and Gynecologist. (1993). Nutrition during pregnancy (Bulletin $\mathrm{n}^{\mathrm{o}}$ 179). Washington, DC: The American College of Obstetrician and Gynecologist, Technical.

American Psychiatric Association. (2000). Diagnostic and statistical manual of mental disorders (4a. ed.). Washington, DC.

Astley, S. \& Clareen, S. K. (2001). Measuring the facial phenotype of individuals with prenatal alcohol exposure: Correlations with brain dysfunctions. Alcohol and Alcoholism, 36, 147-159.

Baron-Cohen, S. \& Bolton, P. (1994). Autism, the facts. New York: Oxford University Press.

Bayés, M., Ramos, J. A., Cormand, B., Hervás, A., Campo, M. \& Duran, E. (2005). Genotipado a gran escala en la investigación del trastorno del espectro autista y el trastorno por déficit de atención con hiperactividad. Revista de Neurología, 40(Supl. 1), 187-190.

Bolton, P. F., Murphy, M., MacDonald, H., Whitlock, B., Pickles, A. \& Rutter, M. (1997). Obstetric complications in autism: Consequences or causes of the condition? Journal of the American Academy of Child and Adolescent Psychiatry, 36(2), 272-281. 
Cook, E. H. (2001). Genetics of Autism. Child and Adolescent Psychiatric Clinics of North America, 10(2), 333-350.

Correa, A., Botto, L., Liu, Y., Mulinare, J. \& Erickson, J. D. (2003). Do multivitamin supplements attenuate the risk for diabetes-associated birth defects? Pediatrics, 111, 1146-1151.

Croen, L. A., Grether, J. K., Hoogstrate, J. \& Selvin, S. (2002). The changing prevalence of autism in California. Journal of Autism and Developmental Disorders, 32(3), 207-215.

Dean, R. S., Gray, J. W. \& Anderson, J. L. (1996). Underlying factor structure of perinatal events. International Journal of Neuroscience, 84, 55-63.

Deykin, E. Y. \& MacMahon, B. (1980). Pregnancy, delivery, and neonatal complications among autistic children. American Journal of Diseases of Children, 134, 860-864.

Faden, V. B., Hanna, E. \& Graubard, B. I. (1997). The effect of positive and negative health behavior during gestation on pregnancy outcome. Journal of Substance Abuse, 9, 63-76.

Finegan, J. \& Quarrington, B. (1979). Pre-, peri, and neonatal factors and infantile autism. Journal of Child Psychology and Psychiatry and Allied Disciplines, 20, 119-128.

Fisher, N. \& Happé, F. (2005). A training study of theory of mind and executive function in children with autistic spectrum disorders. Journal of Autism and Developmental Disorders, 35, 757-771.

Folstein, S. E. (1999). Autism. International Review of Psychiatry, 11, 269-277.

Fraser, A. M., Brockert, J. E. \& Ward, R. H. (1995). Association of young maternal age with adverse reproductive outcomes. The New England Journal of Medicine, 332, 1113-1117.

Gillberg, C. \& Gillberg, I. C. (1983). Infantile autism: A total population study of reduced optimality in the pre-, and neonatal periods. Journal of Autism and Developmental Disorders, 13(2), 153-166.

Gray, J. W., Dean, R. S. \& Rattan, G. (1987). Assessment of perinatal factors. Psychology in the Schools, 24, 15-21.

Gray, J. W., Dean, R. S., Rattan, G. \& Bechtel, B. A. (1988). Mother's self-report of perinatal complications. Journal of Clinical Child Psychology, 17, 242-247.
Huizink, A. C., Robles, P. G., Mulder, E. J. H., Visser, G. H. A. \& Buitelaar, J. K. (2002). Psychological measures of prenatal stress as predictors of infant temperament. Journal of the American Academy of Child Adolescent Psychiatry, 41, 1078-1085.

Iverson, J. M. \& Wozniak, R. H. (2007). Variatin in vocal-motor development in infant siblings of children with Autism. Journal of Autism and Developmental Disorders, 37, 158-170.

Juul-Dam, N., Townsend, J. \& Courchesne, E. (2001). Prenatal, perinatal, and neonatal factors in autism, pervasive developmental disorder-not otherwise specified, and the general population. Pediatrics, 107, 63-68.

Kesmodel, U., Wisborg, K., Olsen, S. F. Brink, T. \& Secher, N. J. (2002). Moderate alcohol intake during pregnancy and the risk of stillbirth and death in the first year of life. American Journal of Epidemiology, 155(4), 305-312.

Lester, B. M., Tronick, E. Z., LaGasse, L., Seifer, R., Bauer, Ch. R., Shankaran, S., et al. (2002). The maternal lifestyle study: Effects of substance exposure during pregnancy on neurodevelopmental outcome in 1-month-old infants. Pediatrics, 110, 1182-1192.

Levy, S., Zoltak, B. \& Saelens, T. A. (1988). Comparison of obstetrical records of autistics and noautistics referrals for psychoeducational evaluations. Journal of Autism and Developmental Disorders, 18(4), 573-581.

Lobel, M. (1994). Conceptualizations, measurement, and effects of prenatal maternal stress on birth outcomes. Journal of Behavioral Medicine, 17, 225-272.

López, S. (2004). Detección de los riesgos maternos perinatales en la etiología de los Trastornos Generalizados del Desarrollo. Disertación doctoral no publicada, Facultad de Psicología, Universidad de Santiago de Compostela, España.

López, S., Rivas, R. M. \& Taboada, E. M. (2008). Los riesgos maternos pre-, peri- y neonatales en una muestra de madres de hijos con trastorno generalizado del desarrollo. Psicothema, 20(4), 684-690.

López, S., Rivas, R. M. \& Taboada, E. M. (2009). Revisiones sobre el autismo. Revista Latinoamericana de Psicología, 41(3), 555-570. 
Lou, H. C., Hansen, D., Nordentoft, M., Pryds, Q., Jensen, F. \& Nim, J. (1994). Prenatal stressors of human life affect foetal brain development. Developmental Medicine and Child Neurology, 36, 826-832.

MacLean, J. E., Szatmari, P., Jones, M. B., Bryson, S. E., Mahoney, W. J., Bartolucci, G., et al. (1999). Familial factors influence level of functioning in pervasive developmental disorders. Journal of the American Academy of Child and Adolescent Psychiatry, 38, 746-753.

Matsuishi, T., Yamashita, Y., Ohtani, Y. Ornitz, E., Kuriya, N., Murakami, Y., et al. (1999). Brief report: Incidence of and the risk factors for autistic disorders in neonatal intensive care unit survivors. Journal of Autism and Developmental Disorders, 29, 161-166.

Orvos, H., Nyirati, I., Hajdú, J., Pal, A. Nyari, T. \& Kovacs, L. (1999). Is adolescent pregnancy associated with adverse perinatal outcome?. Journal of Perinatal Medicine, 27, 199-203.

Pritchard, C. W., Sutherland, H. W. \& Carr-Hill, R. A. (1983). Birthweight and paternal height. British Journal of Obstetrics and Gynaecology, 90, 156-161.

Ribas-Fito, N., Cardo, E., Sala, M., Muga, M. E., Mazon, C., Verdú, A., et al. (2003). Breastfeeding, exposure to organochlorine compounds, and neurodevelopment in infants. Pediatrics, 111, 580-585.

Rivas, R. M., López, S. \& Taboada, E. M. (2009). Etiología del autismo: un tema a debate. Psicología Educativa, 15(2), 107-121.

Saisto, T., Ylikorkala, O. \& Halmesmaki, E. (1999). Factors associated with fear of delivery. Obstetrics \& Gynecology, 94 (5 Pt 1), 679-682.

Shapiro, Ch., Sutija, V. G. \& Bush, J. (2000). Effect of maternal weight gain on infant birth weight. Journal of Perinatal Medicine, 28, 428-431.
Stein, D., Weixman, A., Ring, A. \& Barak, Y. (2006). Obstetric complications in individuals diagnosed with autism and in healthy controls. Comprehensive Psychiatry, 47, 69-75.

Szatmari, P. (2003). The causes of autism spectrum [Editorial]. British Medical Journal, 326, 173-174.

Toth, K., Dawson, G., Meltzoff, A. N., Greenson, J. \& Fein, D. (2007). Early social, imitation play, and language abilities of young non-autistic siblings of children with autism. Journal of Autism and Developmental Disorders, 37, 145-157.

Ventura, S. J., Hamilton, B. E., Mathews, T. J. \& Chandra, A. (2003). Trends and variations in smoking during pregnancy and low birth weight: Evidence from the birth certificate, 1990-2000. Pediatrics, $111,1176-1180$

Wideroe, M., Vik, T., Jacobsen, G. \& Bakketeig, L. S. (2001). Does maternal smoking during pregnancy cause childhood overweight? Paediatric and Perinatal Epidemiology, 17, 171-179.

Wilkerson, D. S., Volpe, A. G., Dean, R. S. \& Titus, J. B. (2002). Perinatal complications as predictors of infantile autism. International Journal of Neuroscience, 112, 1085-1098.

Zwaigenbaum, L., Bryson, E., Rogers, T. S., Roberts, W., Brian, J. \& Szatmari, P. (2005). Behavioral manifestations of autism in the first year of life. International Journal of Developmental Neuroscience, 23, 143-152.

Zwaigenbaum, L., Szatmari, P., Jones, M. B., Bryson, S. E., MacLean, J. E., Mahoney, W. J., et al. (2002). Pregnancy and birth in autism and liability to the broader autism phenotype. Journal of the American Academy of Child and Adolescent Psychiatry, 41(5), 572-579. 
